Title: Assessing the association between changing NRTIs when initiating secondline ART and treatment outcomes

Authors: Julia K. Rohr, $\mathrm{PhD}^{1 *}$, Prudence Ive, $\mathrm{MD}^{2}$, C. Robert Horsburgh, $\mathrm{MD}^{3,4}$, Rebecca Berhanu, $\mathrm{MD}^{5}$, Christopher J. Hoffmann, $\mathrm{MD}^{6,7}$, Robin Wood, $\mathrm{DSc}^{8}$, Andrew Boulle, MBChB ${ }^{9}$, Janet Giddy, $\mathrm{MBChB}^{10}$, Hans Prozesky, $\mathrm{MD}^{11}$, Michael Vinikoor, MD ${ }^{12,13,14}$, Mwanza wa Mwanza, MBChB ${ }^{13}$, Gilles Wandeler, MD ${ }^{15,16}$, Mary-Ann Davies, MBChB, PhD ${ }^{9}$, Matthew P. Fox, DSc $c^{1,4,17}$

\title{
Affiliations:
}

1. Center for Global Health and Development, Boston University, Boston, MA, USA

2. Division of Infectious Diseases, Department of Internal Medicine, Helen Joseph Hospital, Faculty of Health Sciences, University of the Witwatersrand, Johannesburg, South Africa 3. Section of Infectious Diseases, Department of Medicine, Boston Medical Center, Boston, MA, USA

4. Department of Epidemiology, Boston University School of Public Health, Boston, MA, USA

5. Center for Infectious Diseases, University of North Carolina, Chapel Hill, NC, USA

6. Johns Hopkins University School of Medicine and Bloomberg School of Public Health, Baltimore, MD, USA

7. The Aurum Institute, Johannesburg, South Africa

8. Department of Medicine, Desmond Tutu HIV Centre, Institute of Infectious Disease and Molecular Medicine, University of Cape Town, Cape Town, South Africa

9. School of Public Health and Family Medicine, Faculty of Health Sciences, University of Cape Town, Cape Town, South Africa

10. McCord Hospital, Durban, South Africa

11. Division of Infectious Diseases, Department of Medicine, University of Stellenbosch and Tygerberg Academic Hospital, Cape Town, South Africa

12. Department of Medicine, University of Alabama at Birmingham, Birmingham, AL, USA

13. Centre for Infectious Disease Research in Zambia, Lusaka, Zambia

14. School of Medicine, University of Zambia, Lusaka, Zambia

15. Department of Infectious Diseases, Bern University Hospital, University of Bern, Switzerland

16. Institute of Social and Preventive Medicine, University of Bern, Switzerland

17. Health Economics and Epidemiology Research Office, Department of Internal Medicine, School of Clinical Medicine, Faculty of Health Sciences, University of the Witwatersrand, Johannesburg, South Africa

*Current address for correspondence:

Harvard University Center for Population and Development Studies

9 Bow Street, Cambridge, MA 02139 USA

Phone: 617-495-2021

jkrohr@hsph.harvard.edu 


\section{Sources of support:}

The project was supported by Grant Number U01AI069924 from NIH (NIAID, NICHD, NCI, NIDA, NIMH). (PI: Egger and Davies). This study was also made possible by the generous support of the American people through Cooperative Agreement AID 674-A-12-00029 from the United States Agency for International Development (USAID), in partnership with PEPFAR. The contents are the responsibility of the authors and do not necessarily reflect the official views of the NIH, of USAID or the United States Government.

The authors report no conflicts of interest related to this work.

Running head: Switching NRTI in second-line ART

\section{Abstract \\ Background}

After first-line antiretroviral therapy (ART) failure, the importance of change in nucleoside reverse transcriptase inhibitor (NRTI) in second-line is uncertain due to the high potency of protease inhibitors used in second-line.

Setting

We used clinical data from 6,290 adult patients in South Africa and Zambia from the International Epidemiologic Databases to Evaluate AIDS-Southern Africa cohort.

\section{Methods}

We included patients who initiated on standard first-line ART and had evidence of first-line failure. We used propensity score-adjusted Cox proportional hazards models to evaluate the impact of change in NRTI on second-line failure compared to remaining on the same NRTI in 
second-line. In South Africa, where viral load monitoring was available, treatment failure was defined as two consecutive viral loads $>1,000$ copies/mL. In Zambia, it was defined as two consecutive CD4 counts $<100$ cells $/ \mathrm{mm}^{3}$.

\section{Results}

Among patients in South Africa initiated on zidovudine, the adjusted hazard ratio for second-line virologic failure was 0.25 (95\% CI: $0.11,0.57)$ for those switching to tenofovir vs. remaining on zidovudine. Among patients in South Africa initiated on tenofovir, switching to zidovudine in second-line was associated with reduced second-line failure (adjusted hazard ratio $=0.35[95 \%$ CI: $0.13,0.96])$. In Zambia where viral load monitoring was not available, results were less conclusive.

Conclusion

Changing NRTI in second-line was associated with better clinical outcomes in South Africa. Additional clinical trial research regarding second-line NRTI choices for patients initiated on tenofovir or with contraindications to specific NRTIs is needed.

\section{Keywords}

Antiretroviral therapy; South Africa; Zambia; Second-line; nucleoside reverse transcriptase inhibitor; NRTI 


\section{Introduction}

Although drug resistance is rare at antiretroviral therapy (ART) initiation, most patients who fail first-line ART have some resistance to nucleoside reverse transcriptase inhibitors (NRTIs) that are also used in second-line regimens(1-6). In order to optimize the efficacy of NRTIs in second-line, national treatment guidelines in South Africa and Zambia recommend patients change at least one NRTI when switching to second-line(7-11). In practice, changing NRTIs does not always occur, usually due to contraindications to specific NRTIs or lack of availability.

The importance of NRTI activity in second-line ART is questioned due to the high potency of standard protease inhibitors (PIs) in second-line, lopinavir/ritonavir. Second-line ART can successfully suppress HIV in the presence of NRTI drug resistance mutations(12-17), yet the number of NRTIs switched from first- to second-line ART is associated with improved outcomes on second-line(18). We investigated the impact of switching NRTIs on virologic and immunologic outcomes as patients in South Africa and Zambia reach multiple years on secondline.

\section{Methods}

We used data from the International Epidemiologic Databases to Evaluate AIDS Southern Africa (IeDEA-SA) cohort, a National Institutes of Health funded initiative pooling data to address HIV treatment research questions. IeDEA-SA data included medical records from 2004-2013 from 175,933 patients in Zambia and 79,908 patients South Africa, with information on basic patient demographics, height, weight, date of visits, diagnoses, ART drugs, and lab values, including CD4 and, in South African sites, HIV viral load. 
The study population included adult patients $\geq 18$ years, initiated on standard first-line ART (2 NRTIs plus a non-nucleoside reverse transcriptase inhibitor (NNRTI)), and had evidence of first-line failure: either a viral load $>1,000$ copies $/ \mathrm{mL}$ among South African patients after at least six months on treatment, or 2 consecutive CD4 counts $<100$ or a 30\% drop from highest CD4 count among Zambian patients. The first NRTI used in first- and second-line was categorized into zidovudine (AZT), stavudine (d4T), tenofovir (TDF), abacavir (ABC), or other. We evaluated switch to each type of NRTI for patients: (1) initiated on zidovudine, (2) initiated on stavudine, and (3) initiated on tenofovir.

Treatment failure on second-line was our primary outcome and was modeled with crude and propensity score-adjusted Cox proportional hazards regression where propensity score was included in the models as a covariate. Our primary analysis used data from South Africa, where viral load monitoring was available, and treatment failure was defined as two consecutive viral loads $>1,000$ copies $/ \mathrm{mL}$. We also evaluated immunologic failure on second-line ART in Zambia, where treatment failure was persistent CD4 levels below 100 cells $/ \mathrm{mm}^{3}$, defined as two consecutive CD4 counts $<100$ cells $/ \mathrm{mm}^{3}(19,20)$. Potential confounders included year of starting second-line, age, sex, duration on first-line, and first-line NNRTI, as well as CD4 count, viral load, hemoglobin, and creatinine clearance at first-line initiation and at switch to second-line. Multiple imputation was used for missing covariates unless they were missing for $>50 \%$ of patients $(21,22)$. Propensity scores for switching to each type of NRTI versus remaining on the same NRTI were calculated for each model with logistic regression, which allowed us to control for as many confounders as possible while maximizing statistical efficiency(23). 
For South Africa models, propensity scores were adjusted for year of switch to secondline, gender, age, baseline alanine aminotransferase (ALT), hemoglobin, CD4, creatinine clearance, weight, and viral load and CD4 count at time of switch to second-line. Models for changing from tenofovir were not adjusted for year since it was only available in more recent years. For Zambia models, propensity scores were adjusted for year of switch to second-line, gender, age, baseline ALT, CD4, hemoglobin, weight, creatinine clearance, and second-line values of CD4, creatinine clearance, and hemoglobin. Models for changing from stavudine were not adjusted for year because the number initiated on stavudine who remained on stavudine in second-line was too small and propensity score models did not converge when year was adjusted. In sensitivity analyses, we adjusted for loss to follow-up through inverse probability of treatment weighting (24).

\section{Results}

Study sample

In South Africa, 4,614 patients had evidence of first-line virologic failure and switched to a PI-based regimen and in Zambia, 2,061 patients experienced first-line immunologic failure and switched to a PI-based regimen. Patients without visits after date of switch to second-line (2.5\%) and patients who did not have any NRTI identified in the regimen at time of second-line initiation (3.2\%) were excluded. The total sample included 6,290 patients (4,275 in South Africa and 2,015 in Zambia).

Among the 6,290 patients in analysis, the majority were female (61\%) and the median age was 34 years (IQR: 29, 40). A description of the patients is shown by country and by change in NRTI in second-line in Supplemental Digital Content Table 1. 
In both countries, CD4 counts at ART initiation were low (median $<100$ cells/mm ${ }^{3}$ ), and CD4 count at switch to second-line was slightly lower for patients who had a change in second-line NRTI in South Africa, but not in Zambia (Supplemental Digital Content Table 1). Overall, 90\% of patients changed NRTIs at second-line (Table 1). In South Africa, the proportion who changed NRTI in second-line was mostly constant over time (92.5\% in $2004-2006,96.3 \%$ in $2007-2008$, 94.5\% in 2009-2010, and 95.5\% in 2011-2013). In Zambia, the proportion changing NRTIs was lower than in South Africa, and changing NRTI was more common in earlier periods $(85.1 \%$ in $2004-2006,91.8 \%$ in $2007-2008,80.3 \%$ in $2009-2010$, and $72.4 \%$ in $2011-2013$ ).

Follow-up time on second-line was 18 months (IQR: 14-28) for South African patients and 23 months (IQR: 15-33) for Zambian patients. Second-line virologic failure occurred among $15 \%$ of patients in South Africa, and second-line immunologic failure occurred among $7 \%$ of patients in Zambia. Death was recorded for $4 \%$ in South Africa and 2\% in Zambia. On average in South Africa, patients who did not switch NRTIs received slightly more viral load monitoring measurements on second-line (1.6 per year) compared to patients who did switch NRTIs (1.4 per year). Yet in recent years viral load monitoring was done less frequently, with a mean of $>1$ measures per year prior to 2011, and a mean of 1.0 measures per year in 2011 and later.

\section{Second-line Outcomes}

Propensity score-adjusted Cox proportional hazards models for virologic failure in South Africa are shown in Table 2 (summary of propensity scores displayed in Supplemental Digital Content Table 2). Among patients initiated on zidovudine, we observed an association between switching to tenofovir and reduced second-line failure compared to staying on zidovudine 
(adjusted hazard ratio (aHR) 0.25 (95\%CI: 0.11, 0.57). Switching from zidovudine to abacavir was not associated with reduced failure. Among patients initiated on first-line stavudine, there was weak evidence for reduced hazards of failure on second-line associated with switching to tenofovir vs. remaining on stavudine ( $\mathrm{aHR}=0.70$ (95\% CI: 0.42, 1.16)). Switching from stavudine to zidovudine did not have an association with reduced hazards of second-line failure. For patients initiating tenofovir in first-line, follow-up time was more limited because the drug was introduced into South Africa's national program later than stavudine and zidovudine. Changing to zidovudine in second-line vs. remaining on tenofovir was associated with decreased second-line failure (aHR: 0.35; 95\%CI: 0.13, 0.96). While loss to follow-up was common (34\% of patients), weighting models using inverse probability weights to account for loss to follow-up did not impact the hazard ratio point estimates.

Using patient data from Zambia to evaluate second-line immunologic failure showed similar trends, but all hazard ratio estimates had wide confidence intervals (Supplemental Digital Content Table 3).

\section{Discussions}

Among patients in South Africa who failed first-line and switched to second-line ART, change in NRTI was associated with reduced virologic failure on second-line for changes to tenofovir and for change from tenofovir to zidovudine. Changes to other NRTIs had no association with second-line failure. In Zambia, where virologic monitoring was not available and treatment failure on second-line was more poorly defined and likely underestimated(25-28), we did not see strong evidence of a benefit from changing NRTIs in second-line. 
It is possible that drug resistance to NRTIs had an effect on second-line failure, yet previous research has shown that protease inhibitor-based second-line ART can successfully suppress HIV in the presence of NRTI drug resistance mutations and that poor adherence is a more likely cause of second-line failure(12-17,29). Given the importance of adherence, it is possible patients on tenofovir in second-line often had better outcomes because the drug was better tolerated. Interestingly, remaining on tenofovir in second-line after initiating tenofovir in first-line was common in Zambia, perhaps because of physician preference for this drug. This trend may explain why there was less switching of NRTIs in Zambia in recent years as tenofovir became available. We did not see any strong evidence for reduction in second-line immunologic failure associated with switching away from tenofoyir in Zambia. Since most patients now initiate tenofovir-based regimens as first-line ART, further exploration into switching to zidovudine, which was associated with better second-line outcomes, compared to remaining on tenofovir in South Africa, is warranted.

Zidovudine is no longer the preferred NRTI for first-line in Zambia or South Africa, however it may still be used for patients with contraindications to tenofovir (e.g., renal failure). First-line zidovudine in South Africa would typically only have been prescribed over stavudine (under 2004 guidelines) when patients had pre-existing peripheral neuropathy or were at increased risk of hyperlactatemia. Since tenofovir became available in South Africa in 2010, patients normally are only initiated on zidovudine if they have renal failure. In Zambia, zidovudine was a more common option in first-line, and was part of national guidelines before 2010, but has now also been replaced by tenofovir. Our results showed evidence that switching from zidovudine to tenofovir was associated with reduced second-line failure but the impact of switching from zidovudine to abacavir was not clear. For patients initiated on zidovudine-based 
regimens because of renal failure, switching to tenofovir in second-line may not be an option if contraindications remain, and more detailed research into treatment choices for this population is important.

One of the main obstacles in this study is the potential for confounding by indication, which is common in observational studies of drug prescriptions. While propensity scores were used to make groups as comparable as possible with respect to their clinical profile, due to limited data available in medical records, there is likely residual confounding. Patients who stay on the same NRTI because of complications that prevent them from taking certain second-line drugs may have worse outcomes due to these contraindications. Alternatively, patients who switch NRTIs may include more patients who truly failed first-line ART rather than switching regimens for other reasons, and who may have worse outcomes on second-line ART.

Additionally, lack of virologic monitoring in Zambia made it difficult to accurately identify second-line treatment failure and draw more conclusive results from these data. Another potential problem is differential surveillance of patients who remain on the same NRTIs compared to those who switch. We did not see large differences in monitoring between these groups, but monitoring frequency did change over time, along with use of NRTIs in second-line regimens, with modifications to national treatment guidelines, so we controlled for calendar time where possible. Lastly, although we had a large initial sample size, stratification by NRTI used limited the numbers in the models, and with a relatively short follow-up time on second-line, led to some imprecise results.

Our results support that the NRTI in second-line plays a role in second-line outcomes and provide limited evidence in support of current guidelines to change NRTI in second-line, although the impact of NRTI on second-line activity may act through drug resistance, drug side 
effects, or better tolerance of drugs associated with improved adherence. This study supports the need for more research regarding NRTI choices for patients with renal failure who fail zidovudine first-line regimens, ideally with drug resistance data, and more follow-up of patients initiated on tenofovir who must switch to second-line. Observational patient cohorts in South Africa and Zambia are challenging settings for answering these complex questions comparing prescription of different drugs, and more information from clinical trials is necessary.

\section{Acknowledgements}

The project was supported by Grant Number U01AI069924 from NIH (NIAID, NICHD, NCI, NIDA, NIMH) (PI: Egger and Davies). This study was also made possible by the generous support of the American people through Cooperative Agreement AID 674-A-12-00029 from the United States Agency for International Development (USAID), in partnership with PEPFAR. The contents are the responsibility of the authors and do not necessarily reflect the official views of the NIH, USAID, or the United States Government.

\section{List of Supplemental Digital Content}

Supplemental Table 1.doc

Supplemental Table 2.doc

Supplemental Table 3.doc 


\section{References}

1. Wallis CL, Mellors JW, Venter WDF, et al. Varied Patterns of HIV-1 Drug Resistance on Failing First-Line Antiretroviral Therapy in South Africa. J Acquir Immune Defic Syndr. $2010 ; 53: 480-484$.

2. Mansana J, Lessells R, Skingsley A, et al. High-levels of acquired drug resistance in adult patients failing first-line antiretroviral therapy in a rural HIV treatment programme in KwaZulu-Natal, South Africa. PLoS One 2013; 8(8): e72152.

3. Marconi VC, Sunpath H, Lu Z, et al. Prevalence of HIV-1 drug resistance after failure of a first highly active antiretroviral therapy regimen in KwaZulu Natal, South Africa. Clin Infect Dis. 2008; 46(10):1589-97.

4. Van Zyl GU, van der Merwe L, Claassen M, et al. Antiretroviral resistance patterns and factors associated with resistance in adult patients failing NNRTI-based regimens in the Western Cape, South Africa. J Med Virol 2011; 83:1764-9.

5. Wallis C, Aga E, Ribaudo H, et al. Drug susceptibility and resistance mutations after firstline failure in resource limited settings. Clin Infect Dis. 2014; 59(5): 706-715.

6. Hamers RL, Sigaloff KCE, Wensing AM, et al. Patterns of HIV-1 drug resistance after firstline antiretroviral therapy (ART) failure in 6 sub-Saharn African countries: Implications for second line ART strategies. Clinical Infectious Diseases 2012.

7. Republic of South Africa Department of Health. National Antiretroviral Treatment Guidelines 2004.

8. Republic of South Africa Department of Health. The South African Antiretroviral Treatment Guidelines 2010. 
9. Government of the Republic of Zambia. Ministry of Health. National Guidelines on Management and Care for HIV/AIDS. 2004.

10. Government of the Republic of Zambia. Ministry of Health. Antiretroviral therapy for chronic HIV infection in adults and adolescents. New ART Protocols, May 2007.

11. Government of the Republic of Zambia. Ministry of Health. Adult and Adolescent Antiretroviral Therapy Protocols 2010.

12. Dlamini J, Hu Z, Ledwaba J, et al. Genotypic resistance at viral rebound among patients who received lopinavir/ritonavir- or efavirenz-based first antiretroviral therapy in South Africa. J Acquir Immune Defic Syndr. 2011; 58(3):304-308.

13. Ajose O, Mookerjee S, Mills EJ, et al. Treatment outcomes of patients on second-line antiretroviral therapy in resource-limited settings: a systematic review and meta analysis. AIDS 2012; 26.

14. Sigaloff KCE, Hamers RL, Wallis CL, et al. Second-line antiretroviral treatment successfully re-suppresses drug-resistant HIV-1 after first-line failure: prospective cohort in sub-Saharan Africa. Journal of Infectious Diseases 2012.

15. Scherrer A, Boni J, Yerly S, et al. Long-lasting protection of activity of nucleoside reverse transcriptase inhibitors and protease inhibitors (PIs) by boosted PI containing regimens. PLoS One 2012; 7(11):e50307.

16. Levison JH, Orrell C, Gallien S, et al. Virologic failure of protease inhibitor-based secondline antiretroviral therapy without resistance in a large HIV treatment program in South Africa. PLoS One 2012; 7(3):e32144. 
17. Johnston V, Cohen K, Wiesner L, et al. Viral suppression following switch to second-line antiretroviral therapy: associations with nucleoside reverse transcriptase inhibitor resistance and subtherapeutic drug concentrations prior to switch. Jrnl Infect Dis 2014; 209(5):711-20.

18. Pujades-Rodríguez, Balkan, Arnould, et al. Treatment Failure and Mortality Factors in Patients Receiving Second-Line HIV Therapy in Resource-Limited Countries. JAMA 2010; 304(3):303-312.

19. World Health Organization. Antiretroviral therapy for HIV infection in adults and adolescents: Recommendations for a public health approach, 2006. http://www.who.int/hiv/pub/guidelines/artadultguidelines.pdf.

20. Meya D, Spacek LA, Tibenderana H, et al. Development and evaluation of a clinical algorithm to monitor patients on antiretrovirals in resource-limited settings using adherence, clinical and CD4 cell count criteria. JIAS 2009; 12.

21. Von Hippel P. Regression with missing Ys: An improved strategy for analyzing multiply imputed data. Sociological Methodology 2007; 37(1):83-117.

22. Allison, PD. Multiple imputation for missing data: a cautionary tale. Sociological Methods \& Research 2000; 28(3):301-309.

23. Austin PC. An introduction to propensity score methods for reducing the effects of confounding in observational studies. Multivariate Behavioral Research 2011; 46(3): 399424.

24. Cole SR, Hernán MA. Constructing Inverse Probability Weights for Marginal Structural Models. American Journal of Epidemiology. 2008;168(6):656-664. 
25. Moore DM, Awor A, Downing R, et al. CD4+ T-cell count monitoring does not accurately identify HIV infected adults with virologic failure receiving antiretroviral therapy. JAIDS 2008; 49: 477-484.

26. Kanapathipillai R, McGuire M, Mogha R, et al. Benefit of viral load testing for confirmation of immunological failure in HIV patients treated in rural Malawi. Tropical Medicine and International Health 2011; 16(12):1495-1500.

27. Sigaloff KCE, Hamers RL, Wallis CL. Unnecessary Antiretroviral Treatment Switches and Accumulation of HIV Resistance Mutations; Two Arguments for Viral Load Monitoring in Africa. JAIDS 2011; 58(1): 23-31.

28. Keiser O, Tweya H, Boulle A, et al. Switching to second-line antiretroviral therapy in resource-limited settings: comparison of programmes with and without viral load monitoring. AIDS 2009; 23(14):1867-1874.

29. Wallis CL, Mellors JW, Venter WDF, et al. Protease inhibitor resistance is uncommon in HIV-1 subtype subtype C infected patients on failing second-line lopinavir/ $\mathrm{r}$ - containing antiretroviral therapy in South Africa. AIDS Research and Treatment 2011; 769627. 
Table 1. NRTI in second-line regimen stratified by first-line NRTI and country.

\begin{tabular}{llrrrr}
\hline \multirow{2}{*}{$\begin{array}{l}\text { NRTI initiated in } \\
\text { first-line ART }\end{array}$} & $\begin{array}{l}\text { NRTI at } \\
\text { second- }\end{array}$ & \multicolumn{2}{l}{$\begin{array}{l}\text { South Africa } \\
\text { line }\end{array}$} & \multicolumn{3}{c}{ Zambia } \\
& initiation & \multicolumn{1}{l}{ (N, \%) } & & \multicolumn{2}{c}{ (N, \%) } \\
\hline Initiated on AZT & AZT & 74 & 12.5 & 42 & 5.2 \\
& TDF & 253 & 42.8 & 598 & 73.7 \\
& ABC & 185 & 31.3 & 154 & 19 \\
& d4T & 3 & 0.5 & 15 & 1.9 \\
& other & 76 & 12.9 & 2 & 0.3 \\
& TOTAL & 591 & & 811 & \\
\hline Initiated on d4T & d4T & 294 & 9.4 & 37 & 4.5 \\
& TDF & 1088 & 34.8 & 572 & 69.2 \\
& ABC & 64 & 2.1 & 110 & 13.3 \\
& AZT & 1677 & 53.7 & 108 & 13.1 \\
& other & 0 & 0 & 0 & 0 \\
& TOTAL & 3123 & & 827 & \\
\hline Initiated on TDF & TDF & 32 & 5.7 & 124 & 32.9 \\
& AZT & 474 & 84.5 & 138 & 36.6 \\
& ABC & 15 & 2.7 & 81 & 21.5 \\
& d4T & 38 & 6.8 & 34 & 9 \\
& other & 2 & 0.4 & 0 & 0 \\
& TOTAL & 561 & & 377 & \\
\hline
\end{tabular}

AZT = zidovudine; TDF = tenofovir $; A B C=$ abacavir $;$ d4T = stavudine 
Table 2. Hazard ratios and 95\% confidence intervals of second-line virologic failure among South African patients, using Cox proportional hazards models adjusted for propensity scores.

\section{Second-line NRTI}

\begin{tabular}{lllll} 
First-line NRTI & AZT & d4T & TDF & ABC \\
\hline AZT & (ref) & (x) & $0.25(0.11,0.57)$ & $1.08(0.49,2.37)$ \\
\hline d4T & $1.19(0.85,1.68)$ & $($ ref $)$ & $0.70(0.42,1.16)$ & $(x)$ \\
\hline TDF & $0.35(0.13,0.96)$ & (x) & (ref) & $(x)$ \\
\hline
\end{tabular}

$A Z T=$ zidovudine $; \mathrm{d} 4 \mathrm{~T}=$ stavudine; $\mathrm{TDF}=$ tenofovir $; \mathrm{ABC}=$ abacavir ref $=$ Reference category, compared to other estimates in the row $\mathrm{x}=$ Sample size too small 